\title{
An Overview of Vortex Extraction and Visualization in Flow Field
}

\author{
Huaihui WANG ${ }^{a,{ }^{*}}$, Jianliang $\mathrm{CHENG}^{\mathrm{b}}$, Fei WANG ${ }^{\mathrm{c}}$, and $\mathrm{He} \mathrm{ZHANG}^{\mathrm{d}}$ \\ People's Liberation Army of China, 96901 Troops, Beijing, 100094, China \\ aemail: huaihuiwang@nudt.edu.cn, bemail: cz2005cmx@sina.com, 'email: \\ 248744469@qq.com, demail: 1229931030@qq.com, *corresponding author
}

Keywords: Flow Field, Feature Visualization, Vortex

\begin{abstract}
Vortex visualization in flow field plays an important role in scientific research and engineering practice. In recent years, many research achievements have been fulfilled, but there are still several crucial problems to solve. This paper summarized the methods of vortex extraction and visualization in recent years, analyzed the existing problems and the focus of future research.
\end{abstract}

\section{Introduction}

Flow field analysis plays a crucial role in scientific research and engineering, and widely used in many fields such as aircraft design, climate modeling, and so on. As an important means of flow field analysis, flow visualization provides an intuitive expression by mapping data to images, by which effective help is provided for researchers to observe the information contained in data. As the scale of flow field data increases, direct visualization methods faced with the problems of low efficiency of data processing, occlusion in 3D visualization, and so on. In this trend, Feature-based flow field visualization method emerges, and plays a central role in large-scale flow field data visualization. Researchers can quickly locate the important feature in large-scale flow field data with feature visualization, and ignore most data that is repeated or not interested. At the same time, occlusion problem can be effectively relieved, important feature of flow filed be highlighted, and efficiency of researchers be improved with this method.

There are about four kinds of visualization methods, direct flow visualization, texture-based flow visualization, geometric flow visualization and feature-based flow visualization. This classification has been widely recognized in visualization community. In practical applications, researchers usually use a variety of visualization methods to comprehensively analyze the flow field. In recent years, with the development of flow field visualization, more comprehensive visualization methods emerged, such as interactive visualization, parallel and in-situ visualization, and illustrative visualization. This article focuses on the feature-based visualization method and analyzes the research status and existing problems of feature visualization methods.

\section{Definition of Vortex}

There are many features of the flow field, such as vortex, shock wave, separation and attachment line, vector field topology, Lagrangian coherent structures, and so on. Among those flow field feature structures, vortex is one of the most concerned by researchers. Therefore, the feature extraction method for vortex becomes a research hotspot in visualization field. However, vortex has not been defined accurately and generally until now, which also makes the vortex extraction method lack of evaluation criteria. Since the 1970s, many researchers successively proposed their own definition of vortex. The earliest definition of vortex was proposed by Lugt in 1979 [1]. He believes that vortex is the rotation movement of large numbers of particles around a common center. Lugt's vortex definition only qualitatively describes the characteristics of the vortex structure and does not give an accurate, quantitative definition. Subsequently, Hunt proposed the Q-Criterion method [2] in 1987. This method defines vortex zone as a region with a negative $Q$ value, where $Q$ value is the difference between the tension tensor mode and the vorticity mode. This method is widely used in ocean current analysis, but it can cause misdetection. In 1991, Robinson pointed out that when viewed from a 
reference coordinate system moving with a vortex core, the instantaneous streamline is mapped to the normal plane of the vortex line, if the streamline can exhibit a roughly circular or spiral pattern, it can be considered as vortex [3]. Although this definition is more accurate, it has the problem of self-dependence, that is, the definition of vortex depends on the definition of vortex line. In 1997, Portela proposed that vortex consists of central region surrounded by a rotating streamline [4]. This definition also does not accurately specify the nature of vortex features. In 2005, Haller proposed a more objective definition of vortex. He believed that vortex is composed of a collection of flow field trajectories, along which the $\mathrm{M}_{\mathrm{Z}}$ values are uncertain. $\mathrm{M}_{\mathrm{Z}}$ value is the acceleration tensor of tension in the direction of zero tension, indicating the rotation characteristics exhibited by the trajectories [5]. Although this definition provides an objective evaluation criterion, it has not been widely recognized in visualization community. In addition to the above definitions, there are many vortex evaluation standards that will be briefly introduced in the following sections.

\section{Vortex Visualization Methods}

In 2005, Kaufman summarized and classified the classical methods of vortex extraction, as shown in Table 1 [6].

Tab.1. Classical vortex extraction method classification [6]

\begin{tabular}{|l|l|l|l|}
\hline Method & Vortex/ Vortex Core Line & Galilean invariance & Local/ Global \\
\hline Helicity & Vortex Core Line & None-Galilean invariant & Local \\
\hline Swirl Parameter & Vortex & None-Galilean invariant & Local \\
\hline Lambda2 & Vortex & Galilean invariant & Local \\
\hline $\begin{array}{l}\text { Predictor } \\
\text { Corrector }\end{array}$ & Vortex Core Line & Galilean invariant & Global \\
\hline Eigenvector & Vortex Core Line & None-Galilean invariant & Local \\
\hline Parallel Vectors & Vortex Core Line & None-Galilean invariant & Local \\
\hline Maximum Vorticity & Vortex Core Line & Galilean invariant & Local \\
\hline Streamline & Vortex & None-Galilean invariant & Global \\
\hline Combinatorial & Vortex & None-Galilean invariant & Local \\
\hline
\end{tabular}

The vortex/vortex core line in Table 1 refers to whether the feature extraction method is for a vortex region or vortex core line. Galilean invariance refers to the sensitivity of the feature extraction method to the velocity imposed on the overall flow field. For example, all vector on a flow field is superimposed with a velocity in the $\mathrm{X}$ direction, and the feature extraction method is not affected, we call this method Galilean invariant, otherwise it is none-Galilean invariant. Local/global Refers to the data required for the feature extraction method. If a data point can be judged only with the data near it, the method is called a local method. If the entire flow field data is needed, it is called a global method. Here are some of the most important and widely used methods.

Vorticity-Based Vortex Extraction. Vorticity is an important physical quantity in the flow field. Many researchers can get the general situation of the flow field only with vorticity. Therefore, vorticity-based vortex extraction method is widely used in flow field research. Vorticity, also known as curl, represents the maximum degree of rotation at any point in the flow field and the direction of rotation. If $\mathrm{u}(\mathrm{x})=(\mathrm{P}, \mathrm{Q}, \mathrm{R})$ is a vector field defined in three-dimensional space, then the vorticity of the vector field $\mathrm{u}(\mathrm{x})$ at the point $\mathrm{x}$ is:

$$
\boldsymbol{\omega}(\mathbf{x})=\nabla \times \mathbf{u}(\mathbf{x})=\left(\omega_{1}, \omega_{2} \omega_{3}\right)=\left(\frac{\partial \mathrm{R}}{\partial \mathrm{y}}-\frac{\partial \mathrm{Q}}{\partial \mathrm{z}}, \frac{\partial \mathrm{P}}{\partial \mathrm{z}}-\frac{\partial \mathrm{R}}{\partial \mathrm{x}}, \frac{\partial \mathrm{Q}}{\partial \mathrm{x}}-\frac{\partial \mathrm{P}}{\partial \mathrm{y}}\right)
$$


Vorticity field can highlight the position of the vortex in itself. It is generally believed that the extreme point of the vorticity field is the vortex center point. Therefore, Strawn et al. proposed the maximum vorticity method [7] to extract vortex core lines in three-dimensional flow field. This method is finding the points with the largest vorticity value on the surface of the grid and connecting all the points as the vortex core line. Villasenor et al. used vorticity to establish a vortex tube structure [8] and achieved good results. Helgeland et al. studied the method of dynamic vorticity field lines of turbulent data, and interactively analyzed the turbulent data using vorticity field lines through particle advection and seed placement techniques. The visualization results are shown in Figure 1 [9].
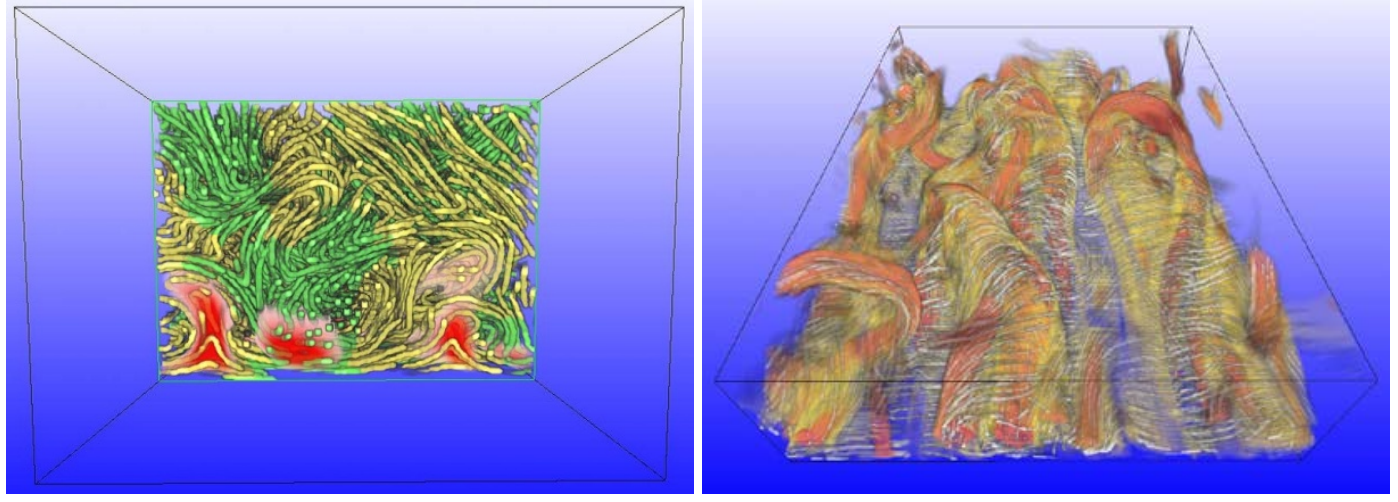

Fig.1. Vortex field line visualization results [9]

Although the vorticity value of vortex region is relatively large, otherwise not. The vorticity-based vortex core extraction method may cause misdetection. However, vorticity-based vortex extraction method is still widely used in ocean current analysis, atmospheric analysis, and aircraft design because of its simplicity, convenient application and physical significance.

Q-Criteria-Based Vortex Extraction. Since Hunt introduced the Q-Criteria [2] in 1987, the Q-Criteria has become the standard method for ocean vortex analysis, and the Q-Criteria in oceanography has also become the Okubo-Weiss standard [10]. $Q$ value is defined as $Q=\varepsilon^{2}-\omega^{2}$, where $\varepsilon$ is the mode of tension tensor and $\omega$ is the mode of vorticity. The most important assumption of the Q-Criteria is that the vorticity value is dominant in the vortex area, that is, the vorticity mode in the vortex area is large and the tension tensor mode is small, but outside the vortex area, the opposite is true. Since the Q-Criteria was proposed, it has been active in ocean current analysis field. In 2011, Williams proposed a self-adaptive vortex extraction method based on the Q-Criteria [11]. This method is based on $\mathrm{Q}$ value and studies the $\mathrm{Q}$ value of the ideal vortex model. The distribution shows that the $\mathrm{Q}$ value has an approximately linear relationship with the square of the radius along the radius. Therefore, Hunt fits Q value and the square of the radius by least square method, and identifies the range of the vortex based on the degree of fitting. This method absorbs the advantages of Q-Criteria and effectively reduces the false detection rate of the Q-Criteria. The visualization results are shown in Figure 2.

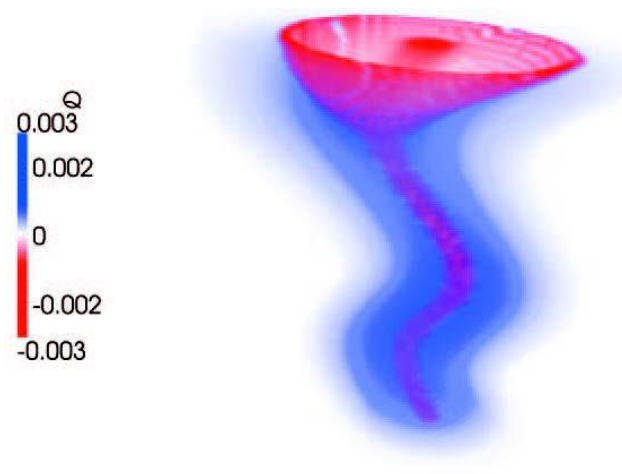

(a)Volume rendering of $Q$ value

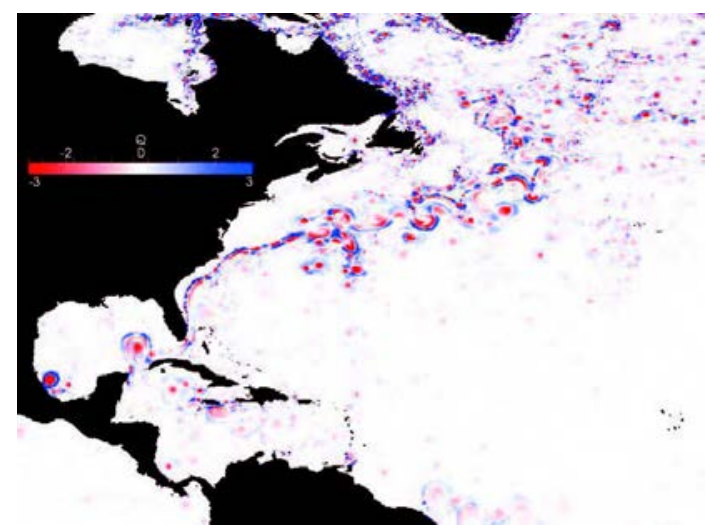

(b) Q value distribution of Atlantic surface

Fig.2. Q-Criteria -based vortex adaptive extraction [11]

$\lambda_{2}$-Based Vortex Extraction. $\lambda_{2}$ [12] method is a commonly used method in aerodynamics. It was proposed by Jeong et al., by which the vortex is defined as the area in the flow field where the 
symmetric matrix $\mathrm{S}^{2}+\Omega^{2}$ contains two negative eigenvalues, where $\mathrm{S}$ is the tension tensor, $\Omega$ is the vorticity tensor. This method is favored by researchers because of its simplicity and practicality. In 2011, Schafhitzel et al. used $\lambda_{2}$ method to study the evolution and interaction of vortices in a three-dimensional time-varying flow field [13]. Koehler et al. studied the visualization of ultra-low Reynolds number vortices around wings of insects during flight [14]. As is shown in Figure 3 shows.
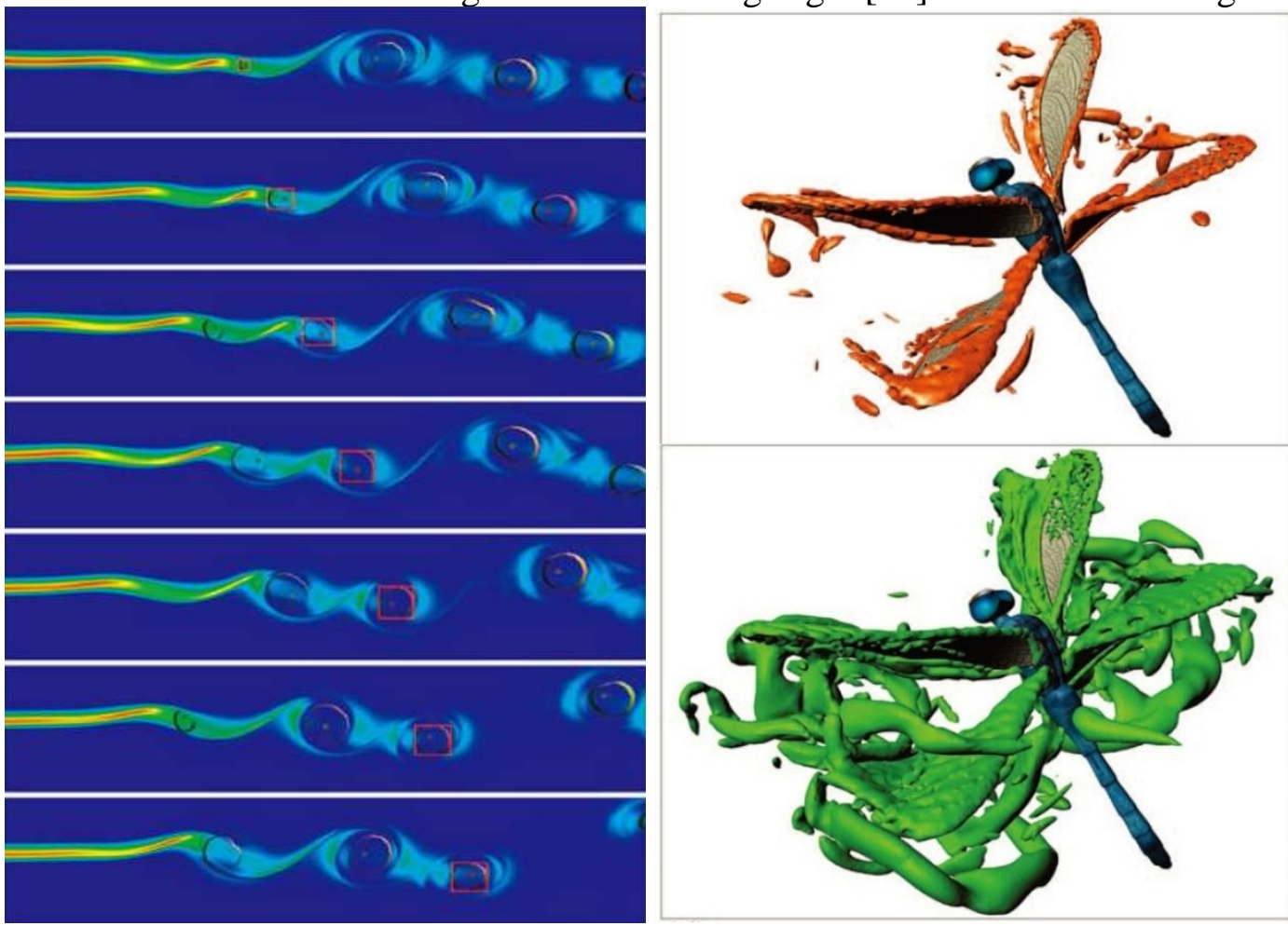

(a) Evolution of vortices [13]

(b) Visualization of the vortices near the wings [14]

Fig.3. Applications of $\lambda 2$ method

The Q-Criteria and $\lambda_{2}$ method are effective in some simple flow field data, but they are not suitable for all flow fields, such as turbulent flow fields that contain strong curved vortex [15]. Therefore, it is very necessary to develop more advanced vortex feature extraction.

Latest Visualization Method of Vortex. In recent years, vortex extraction methods have also made some new developments, and a number of new vortex extraction methods have emerged, among which the following are prominent.

In 2005, Sahner proposed a Galilean-invariant vortex core line extraction method [16]. This method determines the vortex core line by extracting the ridge line of Galilean-invariant physical quantities of the flow field. The physical quantities considered include pressure, vorticity, Q value, $\lambda 2$, and so on. In 2007, Wiebel et al. analyzed boundary induced vortices. They detect vortex by tracking the trajectories of the vortex at the boundary. These trajectories are consist of critical points in the wall shear stress vector field [17]. Weinkauf et al. proposed the method for extracting vortex core line in time-dependent flow field. This method determines the high-dimensional vortex core line by taking the time dimension as a dimension of the data and calculating the high-dimensional traces [18]. In 2008, Fuchs et al. used the parallel vector method to extract vortex of time-varying flow field [19]. The parallel vector is a kind of flow field deriving a vector field, indicating the movement direction of a key data point in a flow field along a certain dimension, in addition, Fuchs also proposed a non-local extended time-dependent vortex detection method based on Euler's standard [20]. In 2009, Petz et al. proposed the idea of a hierarchal vortex region [21] to determine the nested vortices by detecting the closed streamlines in a post-rotational derivation field of a complex two-dimensional flow field. In 2011, Kasten et al. proposed a two-dimensional time-dependent flow field vortex zone detection method based on the acceleration field. This method uses the extremum point of the acceleration field as the vortex core point and the ridge line as the boundary of the vortex area to detect the vortex range [22]. In 2011, Xu Huaxun et al. proposed an approach to the detection of vortex regions based on 
fuzzy theory. This method treats the vortex feature as a fuzzy division in the fuzzy theory and uses the fuzzy C-means clustering method to extract the feature of the flow field [23]. In 2011, Weinkauf et al. proposed a vortex core line detection method based on Stable Feature Flow Fields (SFFF) [24]. From 2012 to 2014, Haller et al. conducted a series of studies of coherent Lagrangian vortices [25-28], discovered the nature of transport barriers around vortices, and compared vortices to the black hole in universe. At the same time, they named this transport barrier as the vortex boundary. The visualization results of several methods are shown in Figure 4.

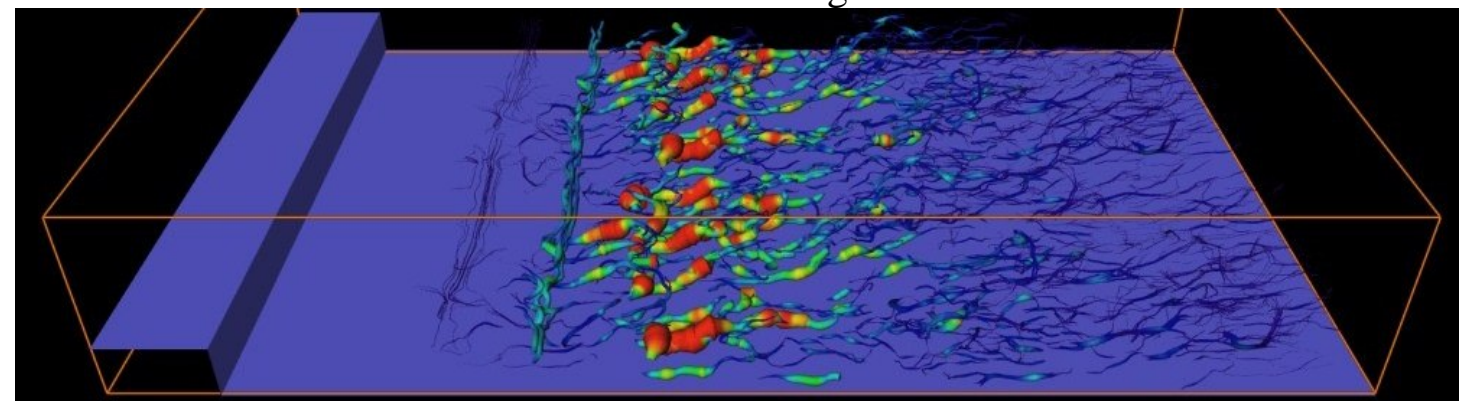

(a) Galilean-invariant vortex core line extraction [16]

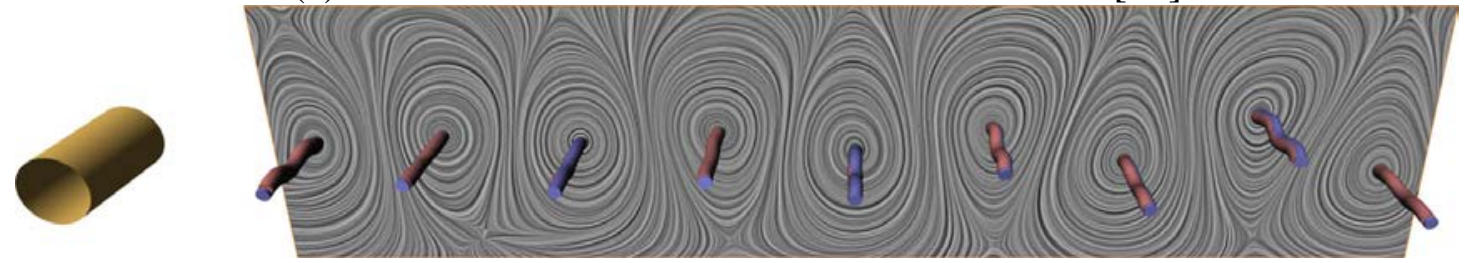

(b) Vortex core line of time-dependent flow field [18]

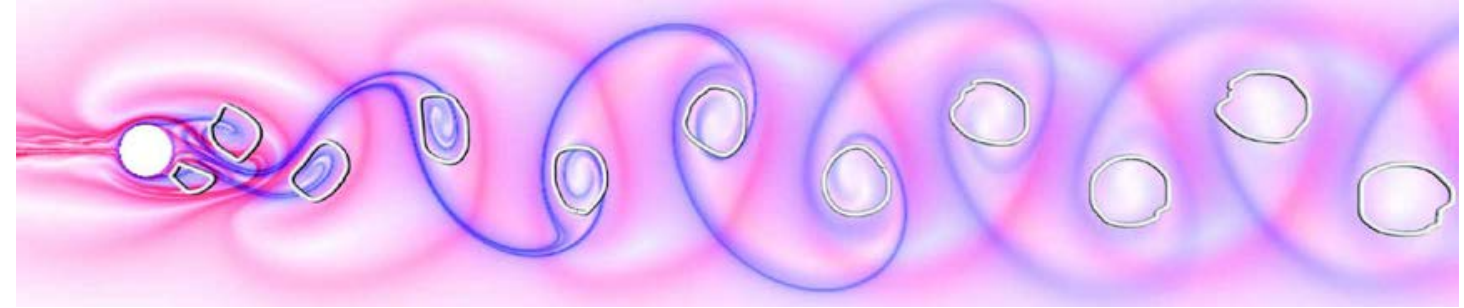

(c) Acceleration field based vortex detection [22]

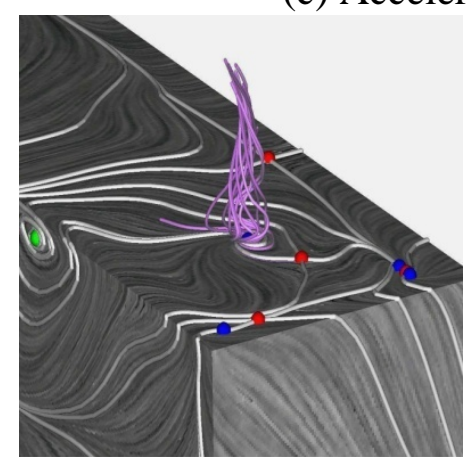

(d) Boundary induced vortex [17]

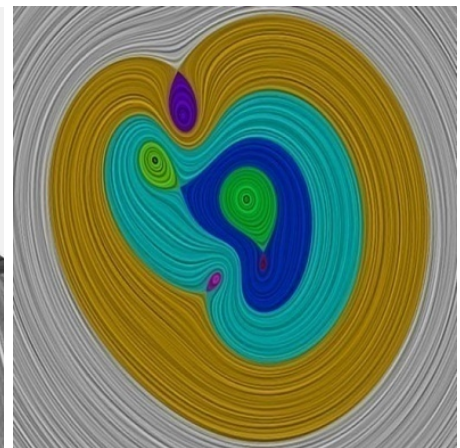

(e) Hierarchal vortex [21]

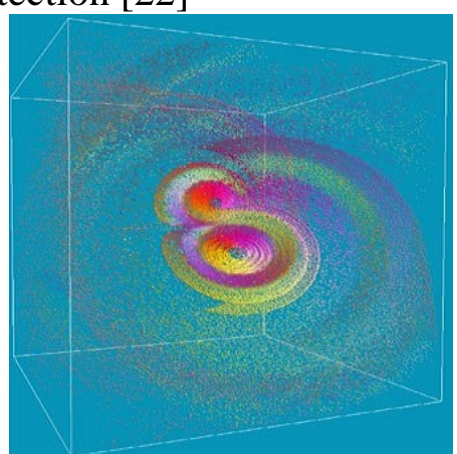

(f) Fuzzy feature extraction [23]

Fig.4. Vortex visualization

Although the definition and extraction method of the vortex have made great progress, it is undeniable that vortex is still one of the most complex and difficult feature structures in the flow field. Although Haller [5, 25-28] gives an objective definition of vortex through rigorous theoretical proof, it is limited to experimental data analysis. However, the various methods mentioned above have poor results when applied to actual complex data. Therefore, it is of great significance to find simple and practical vortex definitions and extraction methods. Next, the current state of research on feature measurement with information measurement will be introduced, especially on vortex feature.

Information entropy is an important physical quantity for measuring information in the information theory. In 1948, Shannon, the father of information theory, first used mathematical language to clarify the quantitative measurement of information and proposed the concept of information entropy. In recent years, with the rapid development of visualization, information 
measurement have plagued many visualization researchers. Therefore, flow information measurement methods based on information entropy began to appear. The application of information entropy in the visual field first appeared in volume rendering [29-31] and later extended to other visualization methods. In 2007, Chan et al. used an extended information entropy as an information measurement for images and light to enhance the image quality of direct volume rendering [32]. In 2010, Xu proposed the concept of flow field information entropy for the first time [33], and successfully expanded information entropy into flow field data to measure flow field information. This method successfully highlights the features of vortices and other high-information structures in the flow field. Based on this method, the distribution of streamlines based on information is studied and the feature structure in the flow field is highlighted. However, this method cannot effectively distinguish the vortex from the saddle because of the lack of consideration of the sampling point position in the definition of the flow field information entropy. Lee et al. studied the viewpoint information and streamline distribution with flow information entropy, and found the best streamline distribution and observation point for observing 3D data fields [34]. Marchesin et al. proposed the concept of streamline information entropy, and distribute the three-dimensional streamline through streamline information measurement to obtain the visualization result with highest information content [35]. Several visualization results are shown in Figure 5.
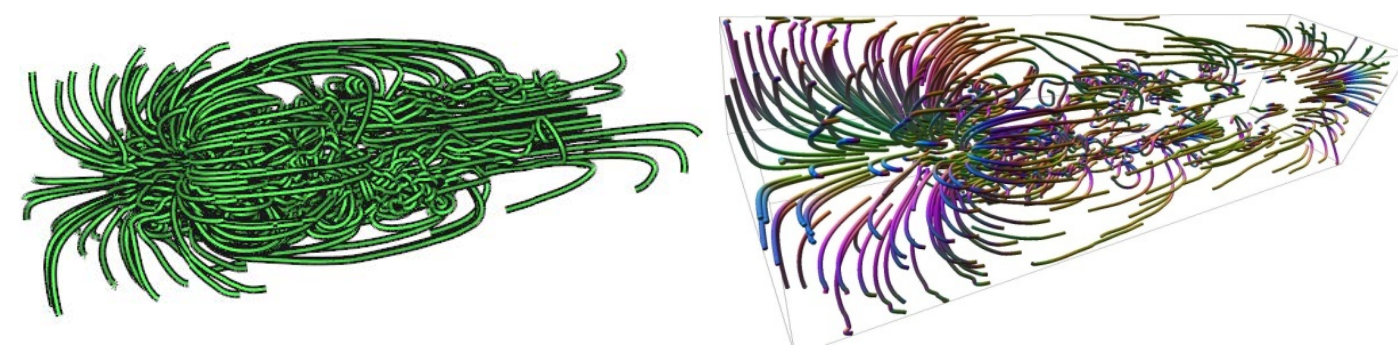

(a) Results with information entropy [34]

(b) Results with streamline information entropy [35]

Fig.5. Visualization results with information entropy

The concept of the flow field information quantity reflects the importance of the quantitative evaluation of information quantity of the flow field. The focus of many flow field evaluation methods is to highlight the feature structure in the flow field. The flow field information entropy represents the flow field information amount. However, the feature structure is not necessarily related to it, so it is very necessary to design an information evaluation system that can represent the flow field feature.

\section{Problems of Visualization}

Feature extraction problems. Feature extraction mainly faces two problems. First of all, it is the question of what is extracted, that is, the problem of the definition of the feature structure. At present, some features that the researchers focus on in the flow field data all lack a clear and well-defined definition, such as vortices, shock waves, separation and attachment line, vector field topology, Lagrange coherence structure (LCS) and so on. Secondly, how to extract features, although many feature extraction algorithms are currently developed, but these algorithms still do not completely solve the key issues such as precise positioning of features, determination of feature ranges, and so on. Developing certain feature extraction algorithms for special field are still the focus of feature visualization study. The feature extraction for two-dimensional and three-dimensional flow fields is the most important application. For example, in the visualization of large-scale two-dimensional data such as global ocean currents, locating the position and range of vortices in data are of great significance.

Feature information measurement problems. Loss of information in visualization of three-dimensional flow field is an inevitable problem. How to measure the amount of information in the flow field and make the visualization result express as much information as possible is an effective way to ensure the quality of visualization results. In recent years, the information measurement of flow field data and its visualization results have attracted the attention of many research experts, and information flow measurement methods based on information entropy have 
obtained certain development, such as flow field information entropy, streamline information entropy and so on, but information entropy measurement in flow field lacks physical significance, and therefore its information volume measurement rules are also lack of persuasion. Therefore, it is especially necessary to design information measurement that can represent the amount of feature structure information.

Time-dependent flow field visualization problems. Most of the flow fields in the objective world are time-dependent flow fields, and steady flow fields only occur under ideal conditions. The researchers generally analyze a certain step in the time-dependent flow field as a steady flow field. This cannot represent the nature of the objective flow field, and many visualization results generated do not exist in the real world, such as streamlines, flow surfaces, and so on. These results can only help researchers understand the overall nature of time-dependent flow fields. Therefore, it is very necessary to study the time-dependent flow field visualization method. However, time-dependent flow field data is usually very large, sometimes hundreds of steps. The current visualization methods for large- scale time-dependent flow field are either with low efficiency or blurred visualization results. How to improve the quality of visualization results while ensuring the efficiency of rendering is the difficulty of visualizing the time-dependent flow field.

In addition to the above-mentioned most common problems, visualization of flow field features also faces other challenges, including unstructured grid data visualization, interactive visualization, time-dependent flow field topology analysis, interpretation of visualization results, verification issues, application of visibility in visualizations, and so on.

\section{Conclusion}

This article briefly reviews some of the important methods for vortex visualization. The classical methods (Q-Criteria, $\lambda_{2}$, etc.) and the more novel methods (flow field information entropy, etc.) are introduced respectively. It can be seen from the foregoing discussion that vortex extraction and information measurement are still worthy of study.

\section{References}

[1] Lugt H J. Vortex flow in nature and technology [M]. New York: Wiley, 1972.

[2] Hunt J C R. Vorticity and vortex dynamics in complex turbulent flows [J]. In Canadian Society for Mechanical Engineering, Transactions. 1987, 11 (1) 21-35.

[3] Robinson S K. Coherent motions in the turbulent boundary layer [J]. Ann. Rev. Fluid Mechanics. 1991, 23 601-639.

[4] Portela L M. Identification and characterization of vortices in the turbulent boundary layer [D]. Stanford: Stanford University, 1997.

[5] Haller G. An objective definition of a vortex [J]. Journal of Fluid Mechanics. 2005, 525 1-26.

[6] Kaufman A, Mueller K. The Visualization Handbook [M]. Oxford UK: Elsevier Inc: Academic Press, 2005.

[7] Strawn R C, Kenwright D N, Ahmad J. Computer visualization of vortex wake systems [J]. AIAA J. 1999, 37 (4) 511-512.

[8] Villasenor J, Vincent A. An algorithm for space recognition and time tracking of vorticity tubes in turbulence [J]. Computer Vision, Graphics, and Image Processing: Image Understanding. 1992, 55 (1) 27-35.

[9] Helgeland A, Reif B A P, Andreassen O, et al. Visualization of Vorticity and Vortices in Wall-Bounded Turbulent Flows [J]. IEEE Transactions on Visualization and Computer Graphics. 2007, 13 (5) 1055-1066. 
[10] Okubo A. Horizontal dispersion of floatable particles in the vicinity of velocity singularities such as convergences [J]. Deep Sea Research and Oceanographic Abstracts. 1970, 17 (3) 445-454.

[11] Williams S, Petersen M, Bremer P-T, et al. Adaptive Extraction and Quantification of Geophysical Vortices [J]. IEEE Transactions on Visualization and Computer Graphics. 2011, 17 (12) 2088-2095.

[12] Jeong J, Hussain F. On the identification of a vortex [J]. J. Fluid Mechanics. 1995, 285 69-94.

[13] Schafhitzel T, Baysal K, Vaaraniemi M, et al. Visualizing the Evolution and Interaction of Vortices and Shear Layers in Time-Dependent 3D Flow [J]. IEEE Transactions on Visualization and Computer Graphics. 2011, 17 (4) 412-425.

[14] Koehler C, Wischgoll T, Dong H B, et al. Vortex Visualization in Ultra Low Reynolds Number Insect Flight [J]. IEEE Transactions on Visualization and Computer Graphics. 2011, 17 (12) 2071-2079.

[15] Roth M, Peikert R. Flow visualization for turbomachinery design [C]. In Proc. Visualization '96. 1996 381-384.

[16] Sahner J, Weinkauf T, Hege H C. Galilean invariant extraction and iconic representation of vortex core lines [C]. In Data Visualization 2005: Proc. of the 7th Joint EUROGRAPHICS - IEEE VGTC Symp. on Visualization(EuroVis 2005). 2005 151-160.

[17] Petz C, Kasten J, Prohaska S, et al. Hierarchical Vortex Regions in Swirling Flow [J]. Computer Graphics Forum. 2009, 28 (3) 863-870.

[18] Weinkauf T, Sahner J, Theisel H, et al. Cores of Swirling Particle Motion in Unsteady Flows [J]. IEEE Transactions on Visualization and Computer Graphics. 2007, 13 (6) 1759-1766.

[19] Fuchs R, Peikert R, Hauser H, et al. Parallel vectors criteria for unsteady flow vortices [J]. IEEE Transactions on Visualization and Computer Graphics. 2008, 14 (3) 615-626.

[20] Fuchs R, Peikert R, Sadlo F, et al. Delocalized Unsteady Vortex Region Detectors [C]. In Proceedings VMV 2008. 2008 81-90.

[21] Petz C, Kasten J, Prohaska S, et al. Hierarchical Vortex Regions in Swirling Flow [J]. Computer Graphics Forum. 2009, 28 (3) 863-870.

[22] Kasten J, Reininghaus J, Hotz I, et al. Two-Dimensional Time-Dependent Vortex Regions Based on the Acceleration Magnitude [J]. IEEE Transactions on Visualization and Computer Graphics. 2011, 17 (12) 2080-2087.

[23] Xu H, Cheng Z, Ralph M, et al. 3D Flow Feature Visualization via Fuzzy Measurement [J]. The Visual Computer. 2011, 27 (6-8) 441-449.

[24] Weinkauf T, Theisel H, Gelder A V, et al. Stable Feature Flow Fields [J]. IEEE Transactions on Visualization and Computer Graphics. 2011, 17 (6) 770-780.

[25] Haller G, Beron-Vera F J. Geodesic theory of transport barriers in two-dimensional flows [J]. Physica D. 2012, 241 1680-1702.

[26] Haller G, Beron-Vera F J. Coherent Lagrangian vortices: The black holes of turbulence [J]. To appear in J. Fluid Mech. 2013.

[27] Farazmand M, Haller G. How coherent are the vortices of two-dimensional turbulence? [J]. To appear. 2014.

[28] Karrasch D, Huhn F, Haller G. Automated detection of coherent Lagrangian vortices in two-dimensional unsteady flows [J]. 2014. 
[29] Gumhold S. Maximum entropy light source placement [C]. In Proceedings of IEEE Visualization. 2002 275-282.

[30] Vazquez P, Feixas M, Sbert M, et al. Viewpoint selection using viewpoint entropy [C]. In Proceedings of the Vision Modeling and Visualization Conference. 2001 273-280.

[31] Bordoloi U, Shen H W. View selection for volume rendering [C]. In Vis'05: Proceedings of the IEEE Visualization 2005. 2005 487-494.

[32] Chan M-Y, Wu Y, Qu H. Quality Enhancement of Direct Volume Rendered Images [C]. In Volume Graphics (The Eurographics Association). 2007 25-32.

[33] Xu L, Lee T-Y, Shen H-W. An Information-Theoretic Framework for Flow Visualization [J]. IEEE Transactions on Visualization and Computer Graphics. 2010, 16 (6) 1216-1224.

[34] Lee T Y, Mishchenko O, Shen H-W, et al. View Point Evaluation and Streamline Filtering for Flow Visualization [C]. In Proceedings of Pacific Visualization Symposium 2011. 2011 83-90.

[35] Marchesin S, Chen C K, Ho C, et al. View-Dependent Streamlines for 3D Vector Fields [J]. IEEE Transactions on Visualization and Computer Graphics. 2010, 16 (6) 1578-1586. 